\title{
Automated Voxel-Wise Brain DTI Analysis of Fitness and Aging
}

\author{
Zhexing Liu ${ }^{1}$, Mahshid Farzinfar ${ }^{*}, 1$, Laurence M. Katz ${ }^{2}$, Hongtu Zhu ${ }^{3}$, Casey B. Goodlett ${ }^{4}$, \\ Guido Gerig ${ }^{4}$, Martin Styner ${ }^{5}$ and Bonita L. Marks ${ }^{6}$ \\ ${ }^{I}$ Department of Psychiatry, University of North Carolina, Chapel Hill, NC, USA \\ ${ }^{2}$ Department of Emergency Medicine and Department of Exercise and Sport Science,University of North Carolina at \\ Chapel Hill, NC,USA \\ ${ }^{3}$ Department of Computer Science University of North Carolina at Chapel Hill, NC, USA \\ ${ }^{4}$ School of Computing University of Utah, Salt Lake City, Utah, USA \\ ${ }^{5}$ Department of Pschiatry and Department of Computer Science University of North Carolina at Chapel Hill, NC, USA \\ ${ }^{6}$ Department of Exercise and Sport Science, Department of Emergency Medicine and Department of Allied Health \\ Sciences, University of North Carolina at Chapel Hill, NC, USA
}

\begin{abstract}
Diffusion Tensor Imaging (DTI) has become a widely used MR modality to investigate white matter integrity in the brain. This paper presents the application of an automated method for voxel-wise group comparisons of DTI images in a study of fitness and aging. The automated processing method consists of 3 steps: 1) preprocessing including image format converting, image quality control, eddy-current and motion artifact correction, skull stripping and tensor image estimation, 2) study-specific unbiased DTI atlas computation via diffeomorphic fluid-based and demons deformable registration and 3) voxel-wise statistical analysis via heterogeneous linear regression and a wild bootstrap technique for correcting for multiple comparisons. Our results show that this fully automated method is suitable for voxel-wise group DTI analysis. Furthermore, in older adults, the results suggest a strong link between reduced fractional anisotropy (FA) values, fitness and aging.
\end{abstract}

Keywords: Diffusion tensor imaging, DTI atlas, voxel-wise analysis, nonlinear warping, aging and aerobic fitness.

\section{INTRODUCTION}

Diffusion Tensor Imaging (DTI) is increasingly being used to investigate the microstructure, integrity and changes of white matter in normal, developing, aging and pathological brains [1-5]. Theoretically, DTI uses a "diffusion coefficient" to quantify the rate and directionality of water displacement in various brain tissues and has been developed as a tool to determine structural integrity of underlying tissues and fiber tracts pathways in white matter [6].

DTI uses a tensor model estimated at each voxel position using linear or nonlinear regression methods given at least 6 gradient directions. Diffusion sensitizing gradients measure signal attenuation of water diffusion by a second order tensor matrix. The most commonly used scalar property maps of DTI are fractional anisotropy (FA) and mean diffusivity (MD), which are calculated from the tensor eigenvalues. Other scalar maps include geodesic anisotropy (GA), parallel/axonal diffusivity (PD/AD), and orthogonal/radial diffusivity $(\mathrm{OD} / \mathrm{RD})$. The principal eigenvector of a tensor determines the overall direction of the fiber structure at the voxel under investigation. DTI fiber tractography can be

*Address correspondence to this author at the Department of Psychiatry, University of North Carolina, Chapel Hill, NC, USA; Tel: +1-919-265 7506; Fax: (919) 966-7659; E-mail: farzinfa@email.unc.edu computed to determine the structures and pathways of major white matter fiber bundles. In this study, we focus on the use of FA images, which is the most commonly employed DTI map in neuroimaging studies. FA measures the degree of diffusion anisotropy and is usually highest in regions of tightly aligned, highly myelinized fiber bundles.

In clinical DTI literature, analytic DTI approaches vary from region-of-interest (ROI) based analysis to voxel based group analysis in neuroimaging studies. ROI analysis typically uses manual or automatically determined ROIs that define white matter structures, followed by statistical analysis of the average tensor indices within those ROIs [7]. ROI methods are probably the most commonly applied method due its simplicity, e.g. for the study of normal development [8-10], schizophrenia [11] or Krabbe's disease [12]. The major drawbacks of ROI analysis suffer from large intra and inter-rater variability and bias in defining meaningful fiber regions, which identify the long and curved shape of fiber bundles. Furthermore, the distribution of DTI property values such as FA within white matter regions is nonGaussian and thus simple averaging over large ROIs is nonoptimal.

In contrast, voxel-wise analysis of DTI is a highly localized analysis methodology that is able to differentiate structures. Voxel-wise analysis has been applied in DTI studies including autism [13] and schizophrenia [14, 15]. These methods are characterized by spatial normalization of a 
population of DTI images, hypothesis testing at each voxel position and multiple comparison corrections. One reason for discrepancies across voxel-wise analysis techniques lies in the difficulty of appropriate anatomical registration of DTI data. This limitation of the established voxel correspondence is pointed out by Chung et al. [16], as they reported superior voxel-wise analysis reliability based on intra-subject registration compared to inter-subject registration. Due to their ease of use, voxel-based methods have become quite common in the last years, especially as a first-line tool for hypothesis generation.

One of the most popular voxel-based analysis frameworks was developed by Smith S.M. et al. [17], called TrackBased Spatial Statistics (TBSS). TBSS brings all the FA images into a specific template with B-spline based nonlinear registration. Registered FA images are then averaged and skeleton of the mean FA images is extracted. Each subjects' aligned FA values are projected to the nearest relevant tract center on the extracted white matter skeleton, where the local maximum FA is computed as the main analysis feature. This procedure compensates for the imprecision in the voxel correspondence and solves the smoothing problems so that statistical analysis can be done on each voxel of the white matter skeleton. In TBSS, only a very small number of voxels in the skeleton are thus tested as compared with a full brain voxel-wise method. The TBSS technique has been used to study preterm infants and adolescent development $[18,19]$, patients with schizophrenia [20], fetal alcohol syndrome [21] and Alzheimer's disease [22]. While this technique reduces the number of statistical tests and simplifies multiple comparison problems, multiple fiber bundles close to intersection areas are likely to be mixed on the skeleton and thus can significantly increase measurement variability. Limited warping quality especially close to the cortical regions due to the large inter-subject variability in brain shape may further limit the utility of TBSS, although this is an issue for most voxel-based analysis methods. In addition, the use of the projected FA maximum values is considered to result in reduced test/retest reliability, as maximal values are more susceptible to noise.

As a complement to DTI voxel-wise analysis, fiber-tract based analysis has been proposed to investigate group differences in DTI [23]. A DTI atlas is created via a fluidbased nonlinear registration method [24] and fiber tracking is performed on the atlas tensor image. The diffusion property profiled are extracted along the parameterized fiber tracts from all the atlas-aligned subjects' tensor images so group comparisons can be performed on these fiber tract diffusion profiles. Several steps in this procedure still need to be performed manually (such as fiber tract source selection), thus limiting the level of automation. While fiber based analysis yield commonly the best compromise between locality and regional average, it is ill-suited for hypothesis generation.

There is great interest in the public health sector to identify biological factors within the central nervous system (CNS) that are associated with cognitive aging and brain structural changes [1,2,25-30]. Epidemiological studies suggest that cardiovascular fitness and education may protect the brain from degradation associated with aging [3134] and Alzheimer's disease [35, 36]. Along these lines, voxel-based morphology (VBM) has been used in high resolution structural MRI images including $\mathrm{T} 1$ and $\mathrm{T} 2$ images [33] and more recently, DTI analysis has been developed to investigate the role of aerobic fitness on white matter integrity in the aged [37-39]. The related studies have found a decline of FA and an increase of MD in several white matter regions in normal aging [1-4].

In this study, we developed a fully automatic methodology for voxel-wise group DTI analysis based on our previous work [40], which requires no manual intervention, while being able to investigate localized changes anywhere in brain. We investigated the relationship between aerobic fitness and white matter integrity in older adults using this methodology. This includes voxel-wise brain white matter group comparisons and voxel-wise correlation studies between DTI properties.

\section{MATERIALS AND METHODS}

\subsection{Subjects and Grouping}

Fifteen older adults between $60-76$ years of age ( 8 males, 7 females) were involved in this study. This study was approved by the University of North Carolina at Chapel Hill's Committee on the Protection of the Rights of Human Subjects. All subjects provided informed written consent for participation. All subjects were college educated, healthy without orthopedic, metabolic, cardiopulmonary, cognitive or MRI scanning limitations. None were on anti-depressants or on medications that would alter the heart rate response to exercise.

Subjects were divided into two groups based on their aerobic fitness according to fitness classifications for men and women aged 60 years and older [41]. Group 1 subjects were aerobically active and reported participating in aerobic exercise for a minimum of $180 \mathrm{~min} / \mathrm{wk}$ for the past 10 years. Group 2 subjects were aerobically inactive and reported participating in aerobic exercise less than $90 \mathrm{~min} / \mathrm{wk}$ over the past 10 years. Aerobic fitness was verified with a physician-supervised, peak exercise (walking) test on a treadmill utilizing Duke's ramped Pepper Treadmill Protocol [42]. The oxygen consumption $\left(\mathrm{VO}_{2}\right.$ peak) was measured using the PARVO TrueMax $\mathrm{VO}_{2}$ Metabolic Cart System (ParvoMedics, Salt Lake City, Utah). The grouping and $\mathrm{VO}_{2}$ peak cut-off values for the groups are listed in Table 1. The gender composition between the two groups were comparable, however there was a significant age difference between them $(\mathrm{p}<0.00001)$.

Whole-head DW Images were acquired with a head only 3.0 Tesla MRI unit (Allegra Siemens Medical Systems) with a maximum gradient strength of $40 \mathrm{mT} / \mathrm{m}$ and a maximum slew rate of $40 \mathrm{mT} / \mathrm{m} / \mathrm{msec}$. A spin-echo echoplanar diffusion tensor weighted sequence $(\mathrm{TE}=79 \mathrm{~ms}, \mathrm{TR}=9200$ $m s$, acquisition matrix $=128 \times 104, \mathrm{FOV}=256 \times$ $208 \mathrm{~mm}^{2}$, slice thickness $=2 \mathrm{~mm}$ and number of slices $=80$ with no gap) was used to acquire the DWI images. A baseline ( $\mathrm{b}=0 \mathrm{~s} / \mathrm{mm}^{2}$, non diffusion-weighted) image and 
Table 1. Aerobic Fitness Classifications per age and Gender. I*Males in the active group had significantly greater $\mathrm{VO}_{2}$ peak values than males in the inactive group $(1-$ Way ANOVA, F $(1,6)=5.73, p=0.05) . * *$ Females in the active group had significantly greater $\mathrm{VO}_{2}$ peak values than females in the inactive group (1Way ANOVA, $F(1,5)=14.51, p=0.01)$, the unit of $\mathrm{VO}_{2}$ peak is $\mathrm{ml} / \mathrm{kg} / \mathrm{min}$

\begin{tabular}{|c|c|c|c|}
\hline $\begin{array}{c}\text { Active Group } \\
\text { (Age: } \mathbf{6 2 . 1 1} \pm \mathbf{2 . 5 7} \text { yrs.) }\end{array}$ & Above Average & Means & (SD) \\
\hline \hline Males $(\mathrm{n}=5)$ & $\mathrm{VO}_{2}$ peak $\geq 33$ & 42.02 & $(7.8)^{*}$ \\
\hline Females $(\mathrm{n}=4)$ & $\mathrm{VO}_{2}$ peak $\geq 26$ & 29.25 & $(4.36)^{* *}$ \\
\hline $\begin{array}{c}\text { Inactive group } \\
\text { age: 72.33 } \pm 2.66 \text { yrs.) }\end{array}$ & Below Average & Means & $(\mathrm{SD})$ \\
\hline Males ( $\mathrm{n}=3)$ & $\mathrm{VO}_{2}$ peak $\leq 32$ & 30.83 & $(1.1)$ \\
\hline Females $(\mathrm{n}=3)$ & $\mathrm{VO}_{2}$ peak $\leq 25$ & 17.6 & $(3.4)$ \\
\hline
\end{tabular}

21 directional DW images $\left(\mathrm{b}=1000 \mathrm{~s} / \mathrm{mm}^{2}\right)$ were acquired $(4 \mathrm{NEX})$ at an isotropic resolution of $2 \times 2 \times 2 \mathrm{~mm}^{3}$

\subsection{Preprocessing}

The preprocessing procedure for our methodology consists of the following steps: DICOM to NRRD format conversion, DWI quality control, skull stripping and DTI computations. The dicom files were first converted into NRRD format ${ }^{1}$ using DicomToNrrdConverter, which is a command line module in Slicer ${ }^{2}$. DWI quality control was performed using DTIPrep [43] $]^{3}$, (see below for more detail). Skull stripping was then conducted using an atlas based tissue segmentation tool called $\mathrm{ABC}$ applied to both the baseline image and the isotropic diffusion weighted image ${ }^{4}$. The tensor images were then estimated using a weighted least squares method [44]. Finally, fractional anisotropy (FA), color coded FA and other property maps were computed straightforwardly from the tensors. DTI estimation and computation was conducted using the DTIProcess toolkit ${ }^{5}$.

\subsubsection{DWI Quality Control}

Artifacts are common in DTI acquisitions. Signal changes produced by severe artifacts result in erroneous diffusion tensor values. Due to the inherent low signal to noise ratio (SNR) and relatively long scanning time of the multi-direction DWI acquisition, compared to common MRI modalities, DWI suffers from eddy-current, head motion and bed vibration artifacts. Even if the artifact in DWI is just a few pixels out of balance, they result in estimation errors of tensors and give confusing artifactual appearances in tensorderived scalar maps and may result in bias throughout DTI analysis. Quality control procedures are of great importance for stable estimation of tensor fields. Currently, most of the quality control procedures are conducted manually by visually checking the DWI data set in a gradient by gradient and slice by slice method. The results often suffer from low

\footnotetext{
${ }^{1}$ http://teem.sourceforge.net/nrrd

${ }^{2}$ http://www.slicer.org

${ }^{3}$ http://www.nitrc.org/projects/dtiprep

${ }^{4}$ http://www.nitrc.org/projects/abc

${ }^{5} \mathrm{http}: / /$ www.nitrc.org/projects/dtiprocess
}

consistency across different data sets because of insufficient QC inter-rater reliability. Our DWI quality control methodology is fully automatic and provides a crucial piece for robust DTI analysis in our study that may reduce the manpower needed for large DTI studies. As far as we know, DTIPrep [43] is the first comprehensive preprocessing tool for DWI quality control with protocoling, reporting and data correction capabilities.

\subsection{DTI Spatial Normalization}

All the DT images were spatially normalized to acquire voxel-wise correspondence across all subjects for meaningful voxel-wise group comparison. DTI spatial normalization was composed of 2 steps. In the first step, we used an unbiased atlas computation method [45] based on nonlinear fluid deformation to initially create an unbiased DTI atlas. In this step, based on our previous work on DTI atlas building [46], we used intensity normalized FA images instead of the curvature of FA image [24] to avoid introducing any bias in subsequent group FA comparison analysis for registration process. Also, intensity normalized FA images outperform curvature FA image in terms of model generalization and specificity in atlas space [46]. The intensity normalized FA images were first smoothed using Gaussian kernel with $\sigma=$ $2 \mathrm{~mm}$, which corresponds to the full width at a half maximum (FWHM) of 4.6. The atlas building procedure was initialized by an affine registration and followed by fluidmodel based nonlinear registration of the intensity normalized FA image which is sensitive to the geometry of white matter, white matter/gray matter and white matter/cerebrospinal fluid (CSF) boundaries. Using the computed deformation field information, we warp each of the individual tensor images into the data specific unbiased space and compute the aligned DT images. After averaging these registered DT images, the first-step DTI atlas was created. Tensor warping and averaging were conducted in a Log-Euclidean space [4749].

To further improve the voxel-wise correspondence quality, we conducted a second mapping step. In this step, we used the pair-wise diffeomorphic demons algorithm [50] to re-map each of the individual DT images to the unbiased atlas created in the first step. The demons algorithm was also initialized with an affine transformation. The DTI transformations were performed the same way as in the first step. After all the DT images were warped, the atlas was updated by averaging the newly warped DT images. In this step, we again used the intensity normalized FA as the driving property map. Fig. (1) shows the schematic view of our novel methodology of the unbiased atlas computation in the first step via applying fluid-based atlas building and the second step after using demons registration algorithm.

Both FA and color coded FA maps show very good smoothness and sharpness within white matter boundaries of the atlas. Structures in the brain stem and pyramidal system are well defined. Overall, visual assessment of the atlas images implies a good alignment among all the subjects. Fig. (2) shows the color coded FA maps of the unbiased atlas created in the first and second mapping step. For the atlas created in the first step, smoothness within most parts of white matter and sharpness at white matter boundaries are obtained, while in some white matter regions, noise-like 


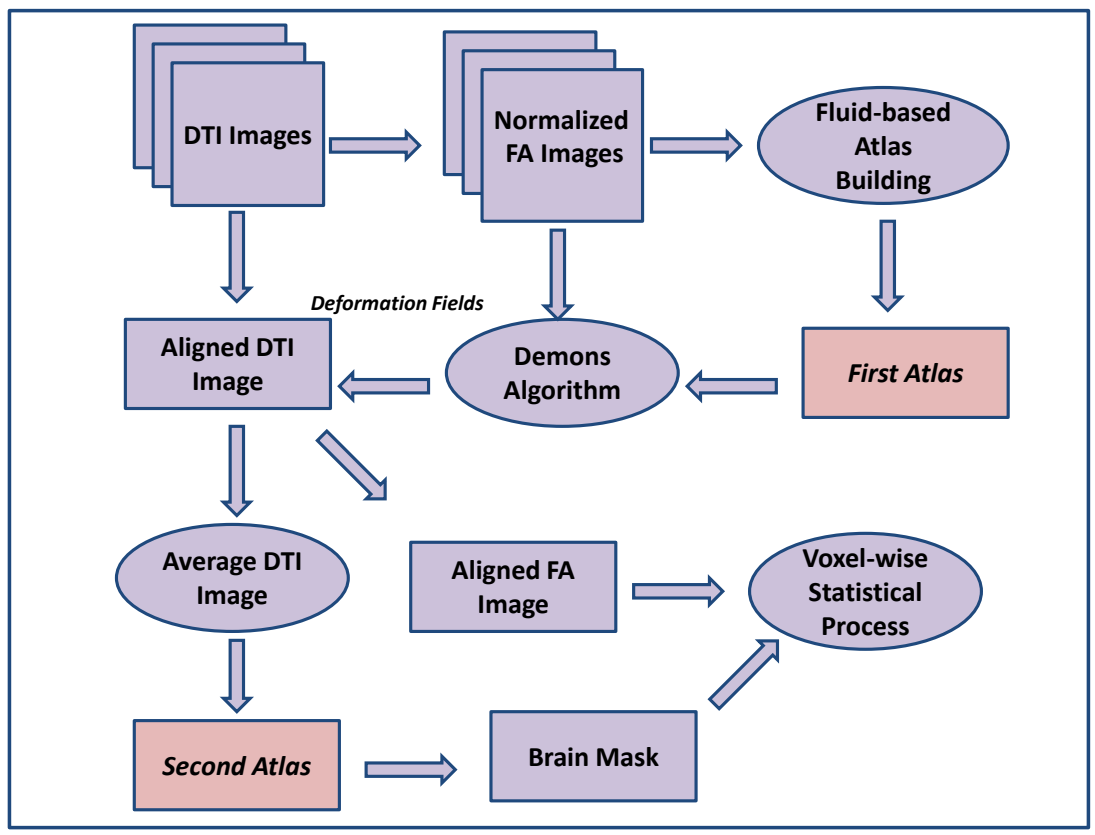

Fig. (1). Scheme of our methodology to building unbiased atlas and applying the voxel-wise statistical analysis.

appearance is still present, especially in the lower part of the cerebellum. This stems from imperfect mapping and errors in the nonlinear deformation field. After a remapping via the diffeomorphic demons algorithm, the white matter shows enhanced smoothness. Also, higher FA values across the whole brain indicate reduced average induced smoothing and thus a better voxel-correspondence. Although the second nonlinear warping step adds complexity and processing time, it improves the quality of voxel correspondence and voxel-wise analysis.

\subsection{Voxel-Wise Group Analysis}

Scalar DTI indices were then calculated from the spatially normalized tensor images. After Gaussian smoothing with $\sigma$ $=2 \mathrm{~mm}$, the images were fed into the statistical comparison

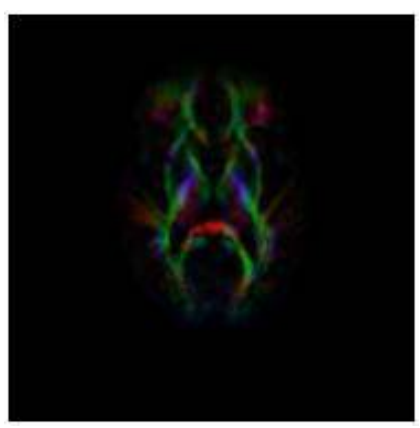

(a)

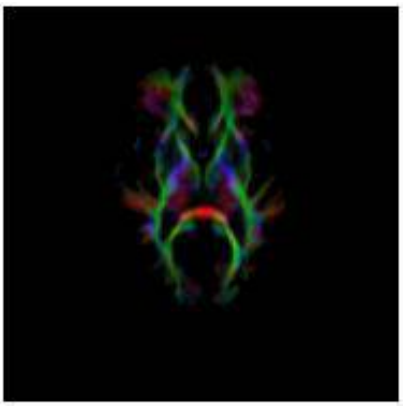

(Ě)

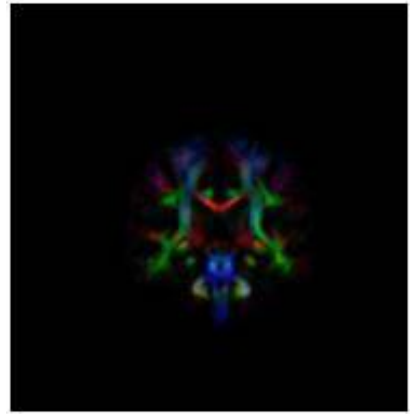

(d)

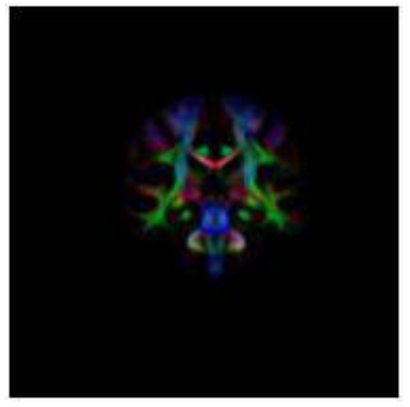

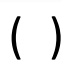

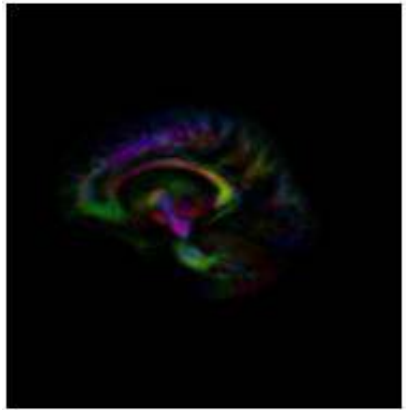

$(\bigoplus)$

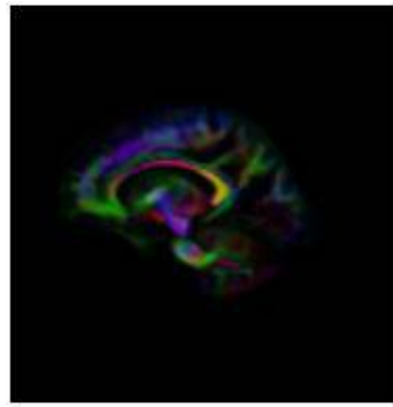

(I)

Fig. (2). Orthogonal views of color coded FA maps of the unbiased DTI atlas created after the rst (a: axial, b: coronal, c: sagittal) and the second (d: axial, e: coronal, f: sagittal) normalization mapping step. Colors encode the principal directions with red $=$ left-right, green $=$ anterior-posterior and blue $=$ inferior-superior direction . 
tool. A white matter mask was created with the atlas FA image using a direct thresholding at $\mathrm{FA}=0.2$ [5]. With this white matter mask, brain regions other than white matter were masked out before the statistical modeling and testing. This reduced not only unnecessary computation but also the number of multiple comparisons, and thus increased statistical power in detecting group differences. For the DTI voxelwise analysis, there is currently no standard statistical framework. Due to the non-Gaussian nature of DTI data, semiparametric statistical methods without assuming any specific parametric distribution are more suitable. Two non-parametric techniques were commonly applied in the DTI setting: one is bootstrap-based testing [51], and the other is permutation-based [16]. Both techniques have shown the ability to derive stable group difference test. In this paper, we used a heteroscedastic linear model for statistical modeling which avoids the limitations of existing statistical analysis of brain differences [52,53] involving the homogeneous variation across subjects and data conforming to Gaussian distribution. We also used a robust test procedure based on wild bootstraping to correct multiple statistical test. The details of the statistical procedures have been explained in detail elsewhere [54]. Fig. (1) shows the voxel-wise group comparison scheme after two-steps DTI atlas building. Note that while doing voxel-wise group analysis, influences of age and gender were corrected as covariants in the heteroscedastic linear model.

\subsection{Voxel-Wise Correlation Analysis}

In aging-fitness studies, $\mathrm{VO}_{2}$ has been shown to be correlated with structural and functional brain image properties $[33,55]$. In this study, we studied the correlations between $\mathrm{VO}_{2}$ peak and DTI properties. It is well known that raw $\mathrm{VO}_{2}$ values demonstrate very large inter-gender and inter-subject variabilities and thus need to be normalized prior/as part of the statistical analysis. In this study, we used a piece-wise linear transformation of gender corresponding measurements from $\mathrm{VO}_{2}$ tables to correct for gender influences. Fig. (3) shows this piece-wise linear transformation. Pearson Correlations were computed between FA values and the corrected $\mathrm{VO}_{2}$ peak values at each voxel position. The significance map of the corrections was also computed using the same testing method as voxel wise group comparison.

\section{RESULTS}

\subsection{Voxel-Wise Group Comparison}

\section{Comparison without Controlling for Age and Gender}

Because of the limited sample size, two group comparisons were performed with and without age and gender corrections. Fig. (4) shows the group comparison analysis results of FA images without correction for age and gender, with the significance mapping overlaid on the atlas FA in violet color $(p<0.05)$. The mean FA is significantly greater

\begin{tabular}{|c|c|c|}
\hline Gender & Raw $\mathrm{VO}_{2}$ & Corrected $\mathrm{VO}_{2}$ \\
\hline \multirow{4}{*}{ Male } & 26.0 & 5.0 \\
& 32.2 & 10.0 \\
& 36.5 & 15.0 \\
& 54.0 & 30.0 \\
\hline \multirow{4}{*}{ Female } & 13.0 & -1.0 \\
& 20.1 & 5.0 \\
& 24.5 & 10.0 \\
& 30.3 & 15.0 \\
\hline
\end{tabular}

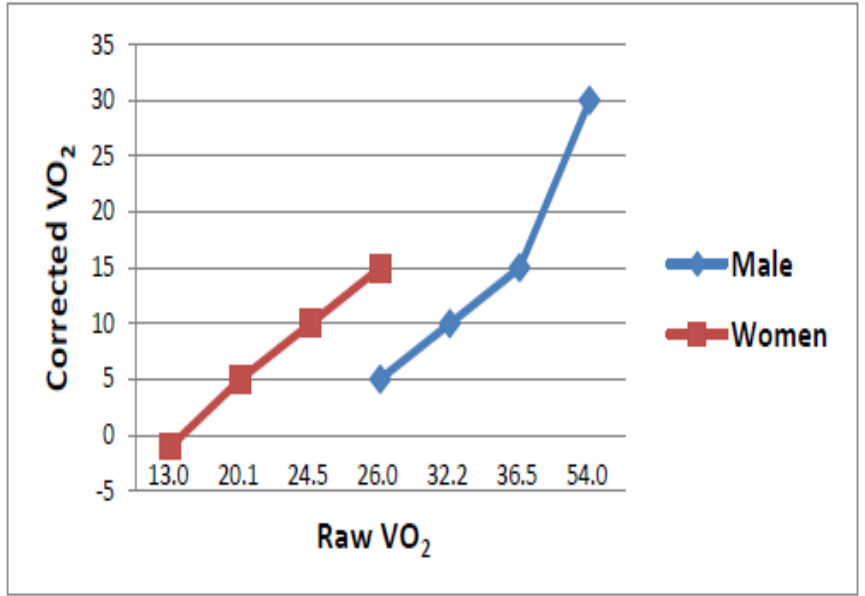

Fig. (3). Gender corresponding $\mathrm{VO}_{2}$ measurements and mapping of the piece-wise linear correction for raw $\mathrm{VO}_{2}$ peak values. 
in the active group (above average aerobic fitness) compared with the inactive group (below average aerobic fitness) primarily in the frontal lobe, parietal lobe, temporal lobe within the regions of cingulum, splenium, arcuate and the corpus callosum (CC) fiber tracts. The occipital lobe has the least number of significant voxels.

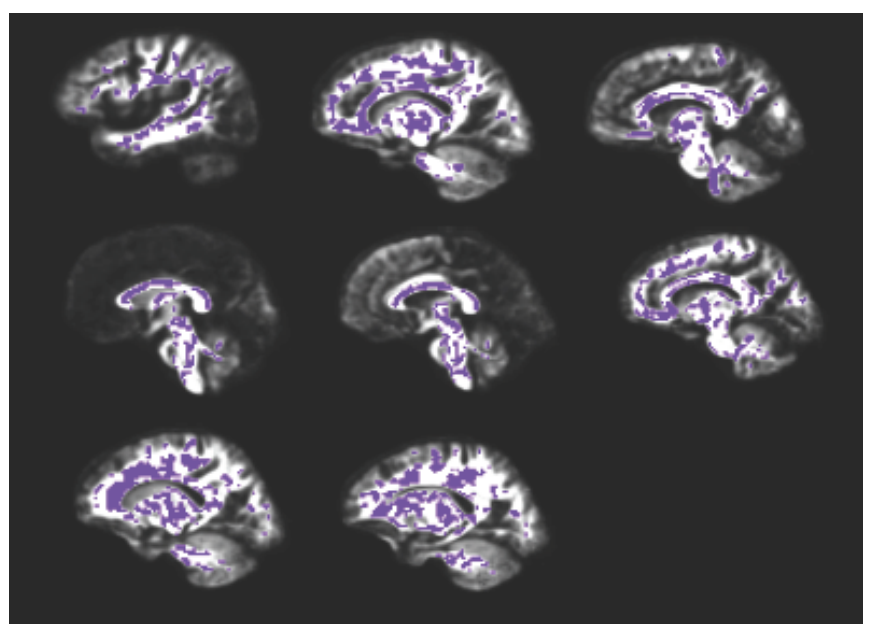

Fig. (4). Voxel-wise group comparison result without correction for age and gender in the sagittal view in right-left order. The significance map is shown in violet where $\mathrm{p}<0.05$.

We compared our group comparison results with the ones from a standard TBSS analysis [17] using the atlas mapped FA images (see Fig. 1). TBSS procedures then generate the mean FA and the tract skeleton, followed by voxel-wise statistics of the maximal FA values projected onto the tract skeleton. The TBSS results are shown in Fig. (5) without correction of age and gender in the datasets. Our own results are in agreement with TBSS results in the sense that significantly different mean FA values are observed in the region of the corpus callosum (CC) and the orbital lobe. However, our approach found significantly larger pattern of differences across the whole brain with $\mathrm{p}<0.05$.

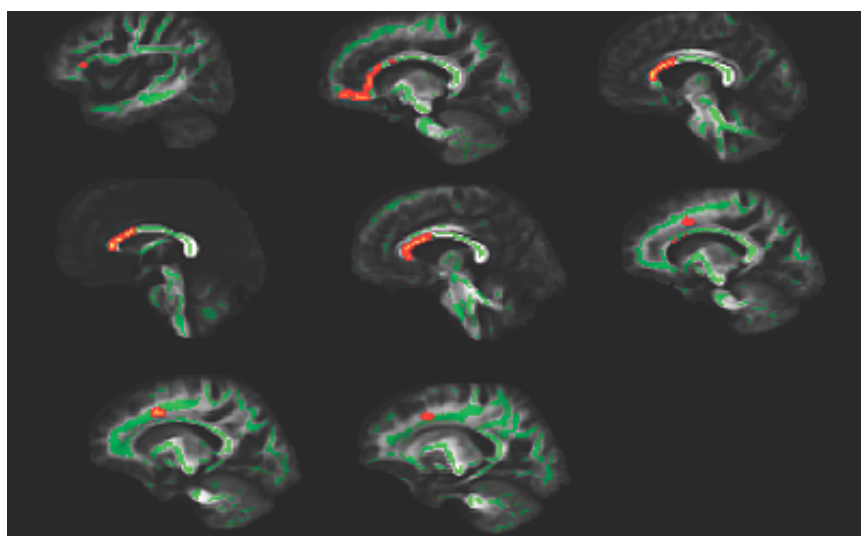

Fig. (5). Voxel-wise group comparison results of applying TBSS approach without age and gender correction. The green areas show the tracts skeleton and the red-yellow areas present the significance map where $\mathrm{p}<0.05$.

An important limitation of the above group comparison results is the cross-sectional design which prohibits us from making casual inference about the effect of fitness on brain structures. Since the average age of the inactive group was greater than active group $(62.11 \pm 2.57) v s .(72.33 \pm 2.66)$, age differences may contribute to some portion of the differences of FA values between the two groups. Moreover, gender has been recognized as an important variable affecting brain studies [56] and associated with related FA differences [57-59]. For example, in gender interaction with the corpus callosum studies, De Bellis et al. [57] observed females had higher FA values in the posterior midbody of the corpus callosum compared to males. Gender differences studies have also focused on human deficiency virus (HIV) associated with white matter alternations [59] and the study of midcingulate cortex and therefore the underlying midcingulum in both hemispheres [60].

\section{Comparison with Controlling for Age and Gender}

The second group comparison analysis results of FA images is shown in Fig. (6) and illustrates controlling for age and gender, with the significance map overlaid on atlas FA in violet color where $p<0.05$. Fig. (6) shows that after correcting for age and gender influences, the majority of significant voxels disappeared, while major regions close to the superior longitudinal fasciculus and the arcuate fasciculus remained.

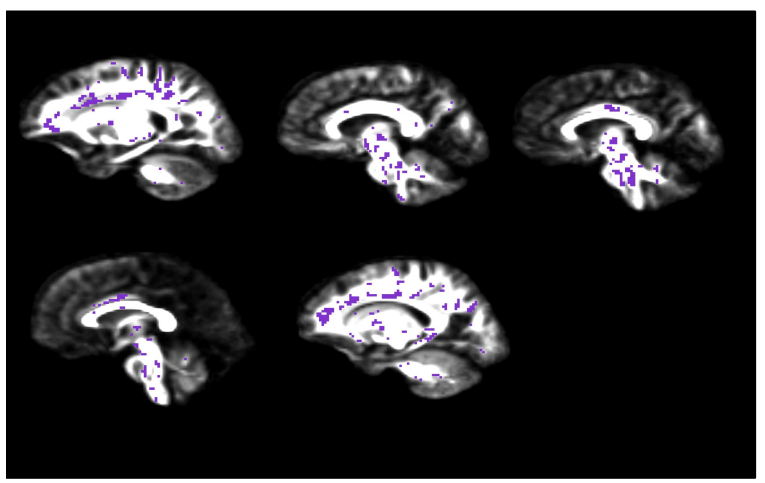

Fig. (6). Voxel-wise group comparison result with correction for age and gender in the sagittal view in right-left order (significance in violet, $\mathrm{p}<0.05)$.

In comparison, no significant locations remained after correction for gender and age via the TBSS approach. Fig. (7) shows the TBSS results that correspond to the five slides shown in Fig. (6). These results confirm the expected higher sensitivity of our wild bootstrapping based voxel-wise statistical approach as forecasted in Zhu et al. [54].

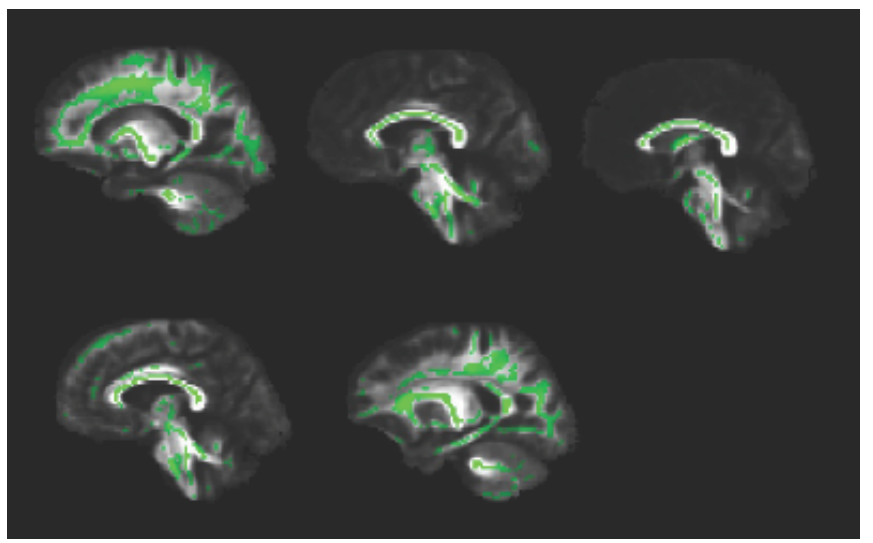

Fig. (7). Voxel-wise group comparison results of applying TBSS approach with age and gender correction. No significant areas are observed. 
Caution should be used when extrapolating the clinical relevance of the results presented here in Fig. (4) and Fig. (6), as well as the differences between Fig. (4) and Fig. (6) since the small sample size has the potential to create unstable results and produce correction errors in the linear model.

\subsection{Voxel Wise Correlation Analysis}

Fig. (8) shows the voxel-wise Pearson correlations between FA and the corrected $\mathrm{VO} 2$ peak values overlaid on a FA map in different views. Only the positive correlations are showed (red) for clarity. The correlation significance was also tested with the same wild bootstrap technique as for group testing. Significantly correlated $(\mathrm{p}<0.05)$ voxels are displayed in violet. In most white matter regions, positive correlations can be seen as expected. Scattered significant voxel can also be seen, with significant correlations between FA and corrected $\mathrm{VO}_{2}$ peak values in the genu, internal capsule and brain stem. As in the previous study, it is noteworthy that these results have been determined on a small sample and thus clinical interpretation should be performed cautiously.

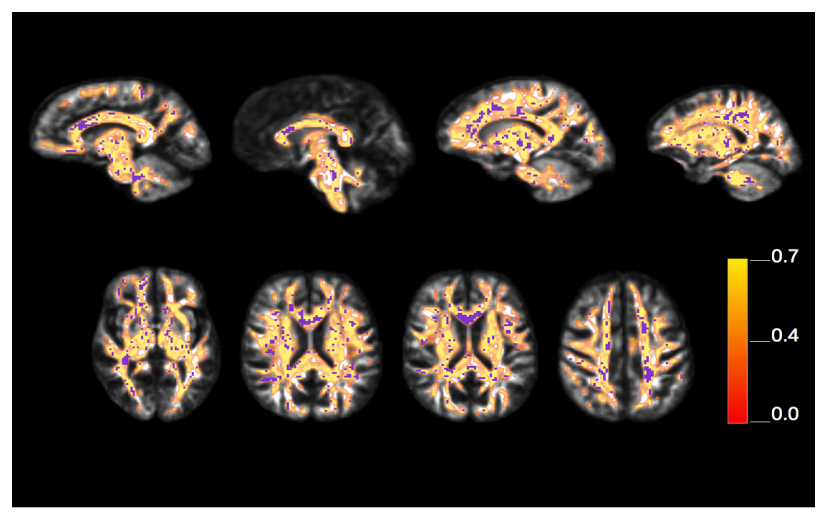

Fig. (8). Voxel-wise correlations between FA values and corrected $\mathrm{VO}_{2}$ peak values in the axial and sagittal views in inferior-superior and right-left orders. Positive correlations are shown in red. The significance map is shown in violet where corrected $\mathrm{p}<0.05$, WarmTint color mapping is used.

\section{DISCUSSION}

\subsection{DTI Normalization and Unbiased Atlas Computing}

Currently, DTI spatial normalization can be classified into 2 categories based on DTI properties driving the matching process: 1) scalar map based [51] and 2) whole tensor based methods. Scalar map based methods have been intensively investigated and well evaluated. These methods can be directly used to estimate the deformation field between DTI images. Whole tensor information based methods are relatively new and lack applications in clinical studies due to the Riemannian/non-Euclidean analysis space for tensors. The overall performances of both methods are comparable at image level, while the whole tensor based methods provide better local tensor corresponding where the orientation information is taken into account. With the development of tensor based registration methods, there is improved matching quality at both image and voxel levels. For spatial nor- malization of group data sets, a well established brain atlas is used as a registration template. All the subjects are transformed into the atlas space and analyzed in that space.

The advantage is the results are presented in a familiar coordinate space to readers, thus it is easier to understand and interpret. The disadvantage is that the warped subjects are biased in shape to the template. Moreover, to map to a standard template, very large deformations may need to be estimated for some subjects, which is one of the major challenges the matching algorithms faces. Compared to a fixed template though, unbiased atlases uses the average space as the center of all the subjects in an unbiased fashion where the overall deformation is minimized. The method is limited in that any change in the data sets will result in a new atlas space. We propose a 2-step normalization strategy, first using the unbiased atlas computing method to build an unbiased atlas, then second, combining the pair-wise registration and average position optimization to enhance warping voxel-wise correspondence.

\subsection{Voxel-Wise Analysis}

Once all the subjects are transformed into atlas space, voxel-wise analysis can be easily conducted for any scalar maps. We presented the FA analysis results in this paper, but it can be applied for any other scalar maps such as MD, $\mathrm{PD}, \mathrm{RD}$, etc. Because we used a heteroscedastic linear model for statistical modeling in this paper, it is very easy to incorporate other factors as co-variants by defining a proper design matrix. This elderly fitness study focused on demonstrating the application of a new automated DTI analysis methodology rather than testing clinical hypotheses related to the fitness parameters. Despite the inherent statistical limitations of sample size and issues of age imbalance, application of this new methodology to this unique population provides some valuable results compared to previous related studies. In order to investigate the effects of fitness exercise on cerebral white matter integrity in older adults, Heo et al. [39] examined a one-year long aerobic training program and determined longitudinal differences in FA values in 70 older adults. They found that improved aerobic fitness is associated with superior white matter integrity specifically in the prefrontal and parietal brain regions. In our paper, while we observed different specificregion effects, we also found similar results with respect to the genu (prefrontal) and cerebrospinal tract (parietal). More related clinical results which corroborate our results can be found in Colcombe et al. [34, 61].

In line with our results, a multiple sclerosis study [35] found a positive association between fitness and the white matter integrity in the right anterior corona radiata. We found similar regions with respect to the cerbrospinal tract (right anterior corona radiata).

An important limitation of our study, as acknowledged before, is most of the group differences were eliminated when age and gender influences were taken into consideration. This may partially be due to the small sample size as well as the difference in mean age between the two groups. The average age for the inactive group was approximately 10 years older than active group. A larger sample size with less age dis-parity between groups may improve the ability to 
detect significant group differences. Furthermore, we are aware of sensitivity limitation in voxel-wise analysis, as we have also observed in this study a null finding in the cingulum region whereas we found a significant correlation with regional analysis in previous work [38].

\section{SUMMARY}

In summary, we describe an automated methodology for voxel-wise group DTI analysis in an older adult population. This methodology is composed of automated preprocessing, normalization and hypothesis testing, which may also be suitable to run on large sample DTI data sets. Our results indicated the improved cardiovascular fitness is associated with mostly prefrontal and parietal brain regions.

\section{CONFLICT OF INTEREST}

The authors declare that they have no conflict of interest.

\section{ACKNOWLEDGEMENTS}

This work was supported by the National Alliance for Medical Image Computing (NAMIC, NIH U54 EB005149); the Autism Centers of Excellence Network at UNC-CH (NIH R01 HD055741), the Neuro developmental Research Center at UNC-CH (NIH P30 HD03110); and the National Institute of Mental Health Conte Center at UNC-CH(MH064065). Research Stimulus Grant from the Biomedical Research Imaging Center (BRIC).

\section{REFERENCES}

[1] Abe O, Yamasue H, Aoki S, et al. Aging in the CNS: comparison of gray/white matter volume and diffusion tensor data. Neurobiol Aging 2008; 29(1): 102 .

[2] Hsu J, Leemans A, Bai C, et al. Gender differences and age-related white matter changes of the human brain: a diffusion tensor imaging study. Neuroimage 2008; 39(2): 566-77.

[3] Pfefferbaum A, Sullivan E, Hedehus M, Adalsteinsson E, Lim K, Moseley M. In vivo detection and functional correlates of white matter microstructural disruption in chronic alcoholism. Alcohol Clin Exp Res 2000; 24(8): 1214-21.

[4] Salat D, Tuch D, Hevelone N, et al. Age-related changes in prefrontal white matter measured by diffusion tensor imaging. Ann NY Acad Sci 2005; 1064(1): 37-49.

[5] Unrath A, Muller H, Riecker A, Ludolph A, Sperfeld A, Kassubek J. Whole brain-based analysis of regional white matter tract alterations in rare motor neuron diseases by diffusion tensor imaging. Hum Brain Mapp 2010; 31(11): 1727-40.

[6] Basser P, Mattiello J, Lebihan D, et al. Estimation of the effective self-diffusion tensor from the NMR spin echo. J Magn Reson 1994; 103(3): 247-54.

[7] Snook L, Plewes C, Beaulieu C. Voxel based versus region of interest analysis in diffusion tensor imaging of neurodevelopment. Neuroimage 2007; 34(1): 243-52.

[8] Bonekamp D, Nagae L, Degaonkar M, et al. Diffusion tensor imaging in children and adolescents: reproducibility, hemispheric, and age-related differences. Neuroimage 2007; 34(2): 733-42.

[9] Gilmore J, Lin W, Corouge I, et al. Early postnatal development of corpus callosum and corticospinal white matter assessed with quantitative tractography. AJNR Am J Neuroradiol 2007; 28(9): 1789.

[10] Hermoye L, Saint-Martin C, Cosnard G, et al. Pediatric diffusion tensor imaging: normal database and observation of the white matter maturation in early childhood. Neuroimage 2006; 29(2): 493-504.
[11] M Kubicki, H Park C, Westin P, et al. DTI and MTR abnormalities in schizophrenia: analysis of white matter integrity. Neuroimage 2005; 26(4): 1109-8.

[12] Guo A, Petrella J, Kurtzberg J, Provenzale J. Evaluation of white matter anisotropy in Krabbe disease with diffusion tensor MR imaging: initial experience1. Radiology 2001; 218(3): 809.

[13] Barnea-Goraly N, Kwon H, Menon V, Eliez S, Lotspeich L, Reiss A. White matter structure in autism: preliminary evidence from diffusion tensor imaging. Biol Psychiatry 2004; 55(3): 323-6.

[14] Burns J, Job D, Bastin M, et al. Structural disconnectivity in schizophrenia: a diffusion tensor magnetic resonance imaging study. Br J Psychiatry 2003; 182: 439-43.

[15] Karlsgodt K, van Erp T, Poldrack R, Bearden C, Nuechterlein K, Cannon T. Diffusion tensor imaging of the superior longitudinal fasciculus and working memory in recent-onset schizophrenia. Biol Psychiatry 2008; 63(5): 512-8.

[16] Chung S, Pelletier D, Sdika M, Lu Y, Berman J, Henry R. Whole brain voxelwise analysis of single-subject serial DTI by permutation testing. Neuroimage 2008; 39(4): 1693-705.

[17] Smith S, Jenkinson M, Johansen-Berg H, et al. Tract-based spatial statistics: voxelwise analysis of multi-subject diffusion data. Neuroimage 2006; 31(4): 1487-505.

[18] Anjari M, Srinivasan L, Allsop J, et al. Diffusion tensor imaging with tract-based spatial statistics reveals local white matter abnormalities in preterm infants. Neuroimage 2007; 35(3): 1021-7.

[19] A Giorgio, K Watkins, Douaud G, et al. Changes in white matter microstructure during adolescence. Neuroimage 2008; 39(1): 5261.

[20] Douaud G, Smith S, Jenkinson M, et al. Anatomically related grey and white matter abnormalities in adolescent-onset schizophrenia. Brain 2007; 130(9): 2375.

[21] Li L, Coles C, Lynch M, Hu X. Voxelwise and skeleton-based region of interest analysis of fetal alcohol syndrome and fetal alcohol spectrum disorders in young adults. Hum Brain Mapp 2009; 30(10): 3265-74.

[22] Stricker N, Schweinsburg B, Delano-Wood L, et al. Decreased white matter integrity in late-myelinating fiber pathways in Alzheimer's disease supports retrogenesis. Neuroimage 2009; 45(1): 10-6.

[23] Goodlett C, Fletcher P, Gilmore J, Gerig G. Group analysis of DTI fiber tract statistics with application to neurodevelopment. Neuroimage 2009; 45(1): S133-42.

[24] Goodlett C, Davis B, Jean R, Gilmore J, Gerig G. Improved correspondence for DTI population studies via unbiased atlas building. Med Image Comput Comput Assist Interv 2006; 9(2): 260-67.

[25] Camara E, Bodammer N, Rodriguez-Fornells A, Tempelmann C. Age-related water diffusion changes in human brain: a voxel-based approach. Neuroimage 2007; 34(4): 1588-99.

[26] Davis S, Dennis N, Buchler N, White L, Madden D, Cabeza R. Assessing the effects of age on long white matter tracts using diffusion tensor tractography. Neuroimage 2009; 46(2): 530-41.

[27] Madden D, Spaniol J, Costello M, et al. Cerebral white matter integrity mediates adult age differences in cognitive performance. $\mathrm{J}$ Cogn Neurosci 2009; 21(2): 289-302.

[28] Takao H, Abe O, Yamasue H, et al. Aging effects on cerebral asymmetry: a voxel-based morphometry and diffusion tensor imaging study. Magn Reson Imgaging 2010; 28(1): 65-9.

[29] Zhang Y, Du A, Hayasaka S, et al. Patterns of age-related water diffusion changes in human brain by concordance and discordance analysis. Neurobiol Aging 2011; 31: 1991-2001.

[30] Eckert M. Slowing down: age-related neurobiological predictors of processing speed. Front Neurosci 2011; 5: 25.

[31] Prakash R, Snook E, Motl R, Kramer A, Aerobic fitness is associated with gray matter volume and white matter integrity in multiple sclerosis. Brain Res 2010; 1341: 41-51.

[32] Erickson K, Prakash R, Voss M, et al. Aerobic fitness is associated with hippocampal volume in elderly humans. Hippocampus 2009; 19(10): 1030-9.

[33] Gordon B, Rykhlevskaia E, Brumback C, et al. Neuroanatomical correlates of aging, cardiopulmonary fitness level, and education. Psychophysiol 2008; 45(5): 825-38.

[34] Colcombe S, Erickson K, Scalf P, et al. Aerobic exercise training increases brain volume in aging humans. J Gerontol A Biol Sci Med Sci 2006; 61(11): 1166. 
[35] Prakash R, Snook E, Motl R, Kramer A. Aerobic fitness is associated with gray matter volume and white matter integrity in multiple sclerosis. Brain Res 2010; 1341: 41-51.

[36] Barnes D, Yaffe K, Satariano W, Tager I. A longitudinal study of cardiorespiratory fitness and cognitive function in healthy older adults. J Am Geriatr Soc 2003; 51(4): 459-65.

[37] Marks B, Madden D, Bucur B, et al. Role of aerobic fitness and aging on cerebral white matter integrity. Ann NY Acad Sci 2007; 1097: 171-4.

[38] Marks B, Katz L, Styner M, Smith J. Aerobic fitness and obesity: relationship to cerebral white matter integrity in the brain of active and sedentary older adults. Br J Sports Med 2011; 45(15): 1208-15.

[39] Susie H. The influence of aerobic fitness on cerebral white matter integrity and cognitive function in older adults: results of a oneyear exercise intervention. Master's thesis, Psychology, University of Illinois at Urbana-Champaign 2010.

[40] Liu Z, Zhu H, Marks B, et al. Voxel-wise group analysis of DTI. ISBI Proceedings of the Sixth IEEE international Conference on Symposium on Biomedical Imaging: Nano to Macro 2009; pp. 80710.

[41] Powers S, Howley E. Exercise physiology: theory and application to fitness and performance. $7^{\text {th }}$ ed. USA: McGraw-Hill 2008; p. 307.

[42] Brown S, Miller W, Eason J. Exercise physiology: basis of human movement in health and disease. Lippincott Williams \& Wilkins 2006.

[43] Liu Z, Wang Y, Gerig G, et al. Quality control of diffusion weighted images. Proc SPIE 2010; 17: 7628.

[44] Goodlett C, Fletcher P, Lin W, Gerig G. Quantification of measurement error in DTI: theoretical predictions and validation. USA: Springer-Verlag 2007; pp. 10-7.

[45] Joshi S, Davis B, Jomier M, Gerig G. Unbiased diffeomorphic atlas construction for computational anatomy. Neuroimage 2004; 23 : S151-60.

[46] Liu Z, Goodlett C, Gerig G, Styner M. Evaluation of DTI property maps as basis of DTI atlas building. Proc SPIE 2010; 7623: 25 .

[47] Arsigny V, Fillard P, Pennec X, Ayache N. Fast and simple calculus on tensors in the Log-Euclidean framework. Med Image Comput Comput Assist Interv 2005; 9: 115-22.

[48] Arsigny V, Fillard P, Pennec X, Ayache N. Log-Euclidean metrics for fast and simple calculus on diffusion tensors. Magn Reson Med 2006; 56 (2): 411-21.
[49] Fillard P, Pennec X, Arsigny V, Ayache N. Clinical DT-MRI estimation, smoothing, and fiber tracking with log-Euclidean metrics. IEEE Trans Med Imaging 2007; 26 (11): 1472-82.

[50] Vercauteren T, Pennec X, Perchant A, Ayache N. Diffeomorphic demons: Efficient non-parametric image registration. Neuroimage 2009; 45(1): S61-S72.

[51] Zhu H, Styner M, Tang N, Liu Z, Lin W, Gilmore J. FRATS: Functional Regression Analysis of DTI Tract Statistics. IEEE Trans Med Imaging 2010; 29 (4): 1039-49.

[52] Nichols T, Hayasaka S. Controlling the familywise error rate in functional neuroimaging: a comparative review. Stat Methods Med Res $2003 ; 12(5): 419$.

[53] Cao J, Worsley K. Applications of random fields in human brain mapping. In: M. Moore Ed. Spatial Statistics: Methodological Aspects and Applications. Springer Lecture Notes in Statistics 2001; vol. 159: pp. 70-182.

[54] Zhu H, Ibrahim J, Tang N, et al. Peterson, A statistical analysis of brain morphology using wild bootstrapping. IEEE Trans Med Imaging 2007; 26(7): 954

[55] Erickson K, Prakash R, Voss M, et al. Aerobic fitness is associated with hippocampal volume in elderly humans. Hippocampus 2009; 19 (10): 1030-9.

[56] Durston S, Hulshoff P, Hilleke E, et al. Anatomical MRI of the developing human brain: what have we learned? J Am Acad Child Adolesc Psychiatry 2001; 40(9): 1012-20.

[57] De Bellis M, Van Voorhees E, Hooper S, et al. Diffusion tensor measures of the corpus callosum in adolescents with adolescen onset alcohol use disorders. Alcohol Clin Exp Res 2008; 32(3): 395-404.

[58] Thatcher D, Pajtek S, Chung T, Terwilliger R, Clark D. Gender differences in the relationship between white matter organization and adolescent substance use disorders. Drug Alcohol Depend 2010; 110(1-2): 55-61

[59] Smith C, Stebbins G, Bartt R, et al. Gender effects on HIVassociated white matter alterations: a voxel-wise DTI study. Brain Imaging Behav 2008; 2(3): 177-91.

[60] Huster R, Westerhausen R, Kreuder F, Schweiger E, Wittling W. Hemispheric and gender related differences in the midcingulum bundle: A DTI study. Human Brain Mapp 2009; 30(2): 383-91.

[61] Colcombe S, Erickson K, Raz N, et al. Aerobic fitness reduces brain tissue loss in aging humans. J Gerontol A Biol Sci Med Sci 2003; 58(2): M176.

(C) Liu et al.; Licensee Bentham Open.

This is an open access article licensed under the terms of the Creative Commons Attribution Non-Commercial License (http://creativecommons.org/ licenses/by-nc/3.0/), which permits unrestricted, non-commercial use, distribution and reproduction in any medium, provided the work is properly cited. 